\title{
Anchos Mesiodistales de Dentición Temporal en Niños de la Ciudad de Concepción, Chile y su Correlación con el Tamaño de Coronas Metálicas Preformadas de Stock
}

\author{
Mesiodistal Width in Temporary Molars of Chilean Children of Concepción \\ and his Correlation with Standard Preformed Steel Crowns
}

María Francisa Torres Chianale*; Lucía Lorena Bravo Rivera*; Claudia Fierro Monti* \& María Antonieta Pérez Flores ${ }^{* *}$

TORRES, C. M. F.: BRAVO, R. L. L.: FIERRO, M. C. \& PÉREZ, F. M. A. Anchos mesiodistales de dentición temporal en niños de la ciudad de Concepción, Chile y su correlación con el tamaño de coronas metálicas preformadas de stock. Int. J. Odontostomat., 4(1):53-57, 2010.

RESUMEN: Las coronas metálicas preformadas son el tratamiento de elección al restaurar extensas caries en molares temporales. Para lograr un óptimo tratamiento es necesario conocer los anchos mesiodistales de las piezas involucradas en la terapia restauradora. En nuestro medio se utilizan los anchos mesiodistales de otras poblaciones, al conocer que pueden existir diferencias significativas con nuestra población en particular decidimos estudiar el ancho mesiodistal en nuestros pacientes. El propósito de esta investigación fue determinar anchos mesiodistales de molares temporales sanos en niños chilenos, analizando dimorfismo sexual, simetría intraarcada y con la pieza contralateral, así como la concordancia con los tamaños estandarizados de las coronas metálicas preformadas. Se realizó un estudio descriptivo odontométrico, seleccionando 34 niños (17 hombres y 17 mujeres) que asistían a la Clínica de Odontopediatría. Se procedió a medir anchos mesiodistales con un calibre ortodóncico en modelos de yeso obtenidos de cada paciente. Simultáneamente se midieron anchos mesiodistales de coronas metálicas preformadas. No se observan diferencias estadísticamente significativas entre los diámetros de piezas derechas e izquierdas ni entre géneros. Molares inferiores presentaron mayor ancho mesiodistal que los superiores. El promedio de ancho mesiodistal de molares temporales se corresponde de manera significativa con un solo tamaño de corona metálica preformada, salvo en las piezas 5.5 y 8.5 donde la correlación es con 2 tamaños de coronas metálicas.

PALABRAS CLAVE: anchos mesiodistales, dientes temporales, coronas preformadas.

\section{INTRODUCCIÓN}

El análisis de la dentición primaria es importante para reconocer, diagnosticar, corregir problemas oclusales y planificar tratamientos restauradores en todos los estados del desarrollo dentario.

Al enfrentarnos con pacientes con extensas destrucciones coronarias la planificación de nuestro tratamiento apunta a la realización de coronas metálicas (Ministerio de Salud, 2005; Randall, 2002). Para desarrollar esta terapia, conocer los anchos mesiodistales de las coronas de las piezas temporales es esencial, utilizando normalmente datos de niños blancos americanos, los que no siempre son representativos ya que pueden existir variaciones étnicas (Jensen et al., 1957).
El objetivo de este trabajo es poder determinar el ancho mesiodistal de molares temporales sanos en una población chilena analizando dimorfismo sexual, simetría intraarcada y con la pieza contralateral, así como la concordancia entre los tamaños estandarizados de la coronas preformadas y los tamaños de los molares obtenidos en este estudio para determinar cuáles son los tamaños de coronas más utilizadas en pacientes infantiles chilenos, lo que podría disminuir el costo adquisitivo a nivel de servicios odontológicos , adquiriendo sólo los tamaños más concordantes de coronas metálicas y de este modo masificar esta terapia restauradora.

Instructor Facultad de Odontología Universidad de Concepción, Chile.

** Profesor Asistente Facultad de Odontología Universidad de Concepción, Chile.

*** Profesor Asociado Facultad de Odontología Universidad de Concepción, Chile. 
TORRES, C. M. F.: BRAVO, R. L. L.: FIERRO, M. C. \& PÉREZ, F. M. A. Anchos mesiodistales de dentición temporal en niños de la ciudad de Concepción, Chile y su correlación con el tamaño de coronas metálicas preformadas de stock. Int. J. Odontostomat., 4(1):53-57, 2010.

\section{MATERIAL Y MÉTODO}

Se diseñó un estudio descriptivo odontométrico en niños preescolares que acudieron a solicitar tratamiento odontopediátrico a la Facultad de Odontología de la Universidad de Concepción. Se seleccionaron 34 niños (17 hombres y 17 mujeres) con dentición temporal pura. Para ingresar al estudio, los menores debieron cumplir los siguientes criterios de inclusión: No presentar desarmonías faciales aparentes, tener molares temporales con erupción completa, no tener piezas permanentes en boca, tener una oclusión aceptable, no tener caries ni restauraciones proximales y no presentar ningún síndrome.

Se excluyó a todos los menores cuyos modelos presentaban imperfecciones técnicas que impidieran la correcta medición del diente.

Se tomaron impresiones con alginato Jeltrate rápido de cada arco y se realizó el vaciado de modelos de estudio en yeso Moldano en los cuales se procedió a realizar las mediciones de 272 molares temporales. Para la comparación se utilizaron las coronas preformadas de acero inoxidable para molares temporales de $3 \mathrm{M}$ ESPE.

Las mediciones fueron hechas por un solo operador, quien realizó dos mediciones, obteniendo así un promedio de ambas medidas que correspondió a la medida final. Si las medidas diferían en más de $0.25 \mathrm{~mm}$ se tomaba una tercera medida y las dos más próximas se promediaron, obteniéndose la medida final. Se utilizó un calibre ortodóncico Vernier de puntas finas marca Dentaurum, modelo 042-751 Münchner (de una precisión de décima de milímetro). Las mediciones fueron realizadas con luz artificial, utilizando el criterio de medición de acuerdo con Moorrees \& Reed (1964) como la máxima distancia entre los puntos de contacto mesial y distal; poniendo el eje paralelo a las superficies oclusales.

Para determinar el tamaño de las coronas metálicas se realizó la misma medición que en los molares. Comprobando el tamaño de corona preformada correspondiente al ancho mesiodistal del molar, para determinar qué número de corona preformada tiene mayor correlación con el ancho mesiodistal de los molares temporales.

Los datos obtenidos de las mediciones fueron analizados con el paquete estadístico SPSS. Calculando promedios y desviación estándar de cada dimensión dentaria y se comprobó normalidad de las variables a través del test de bondad de ajuste de KolmogorovSmirnov. Se utilizó T-test para muestras independientes para comprobar diferencias entre los anchos mesiodistales de molares temporales contralaterales, con arcos superior e inferior y por sexo y T-test para muestras relacionadas para verificar el grado de correspondencia entre anchos mesiodistales de molares temporales con coronas metálicas preformadas. Se consideraron diferencias significativas con un $p<0,05$.

\section{RESULTADOS}

No se observaron diferencias estadísticamente significativas $(p>0,05)$ al comparar molares temporales contralaterales, según se aprecia en la Fig. 1.

Al comparar molares temporales entre arcadas superior e inferior se encontraron diferencias estadísticamente significativas $(p<0,0001)$ siendo las piezas del arco inferior de mayor tamaño que las del arco superior (Fig. 2).

Al analizar dimorfismo sexual no se encontraron diferencias estadísticamente significativas $(p>0,05)$ por lo que los hombres y las mujeres de esta serie presentaron anchos mesiodistales de molares temporales de similar magnitud (Fig. 3).

Al comparar promedios de cada molar temporal con las medidas estándar de las coronas metálicas preformadas se encontró que la mayoría de las piezas se corresponden adecuadamente con una única corona preformada, salvo en las piezas 5.5 y 8.5 en donde fue posible encontrar dos coronas preformadas que se adecuaron a las dimensiones de la pieza dentaria original (Tabla I).

Tabla I. Correspondencia entre anchos mesiodistales de molares temporales con coronas metálicas preformadas.

\begin{tabular}{cc}
\hline Pieza Dentaria & $\mathrm{N}^{\circ}$ de Corona Metálica \\
\hline 5.5 & $2-3$ \\
5.4 & 2 \\
6.5 & 2 \\
6.4 & 3 \\
7.5 & 5 \\
7.4 & 4 \\
8.5 & $4-5$ \\
8.4 & 4 \\
\hline
\end{tabular}




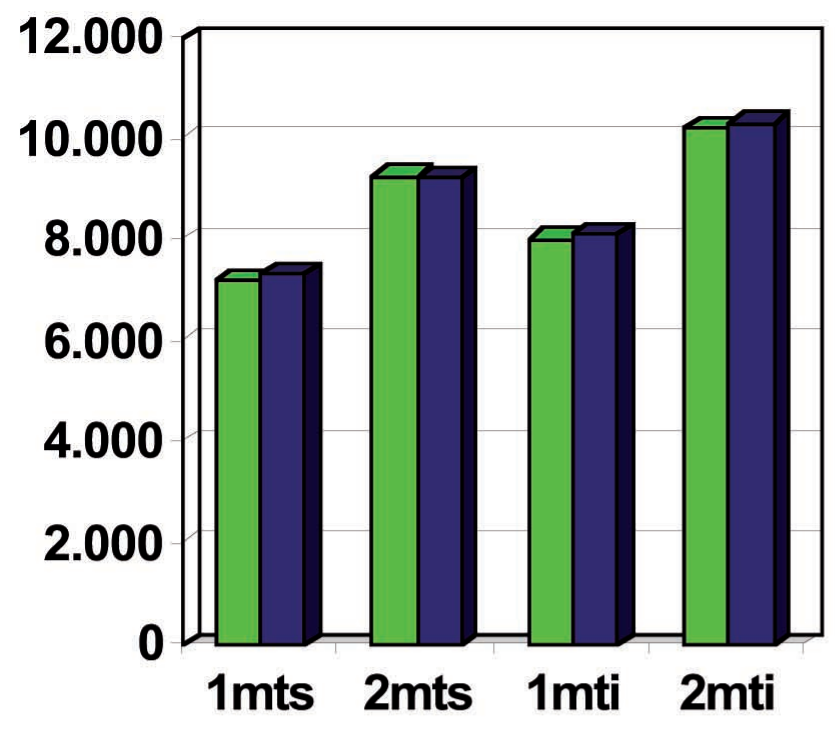

Fig. 1. Comparación entre anchos mesiodistales de molares temporales contraletarales.

\section{$\square$ Lado derecho \\ u Lado izquierdo}

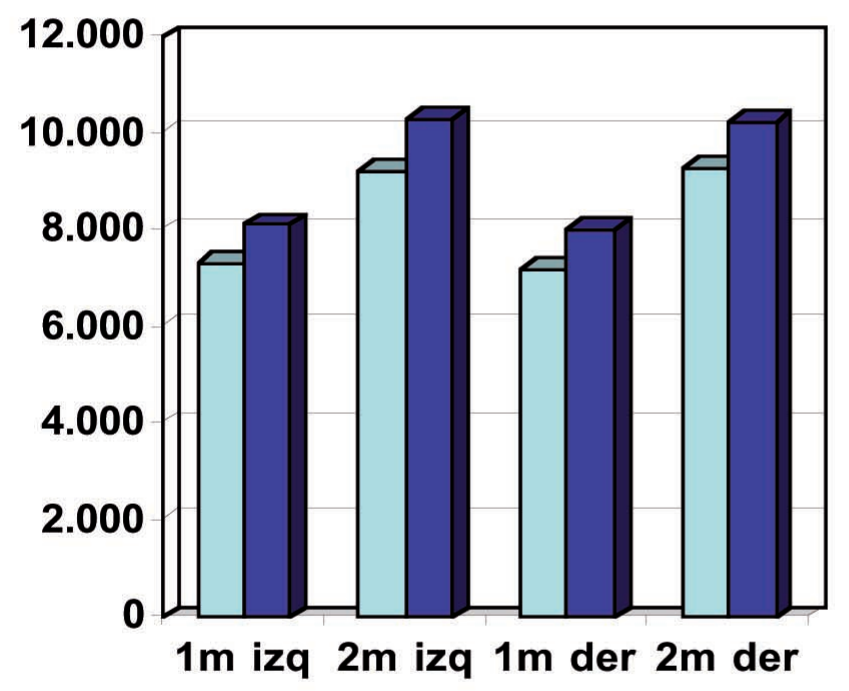

Fig. 2. Comparación ente anchos mesiodistales entre molares temporales de arcos superior e inferior.

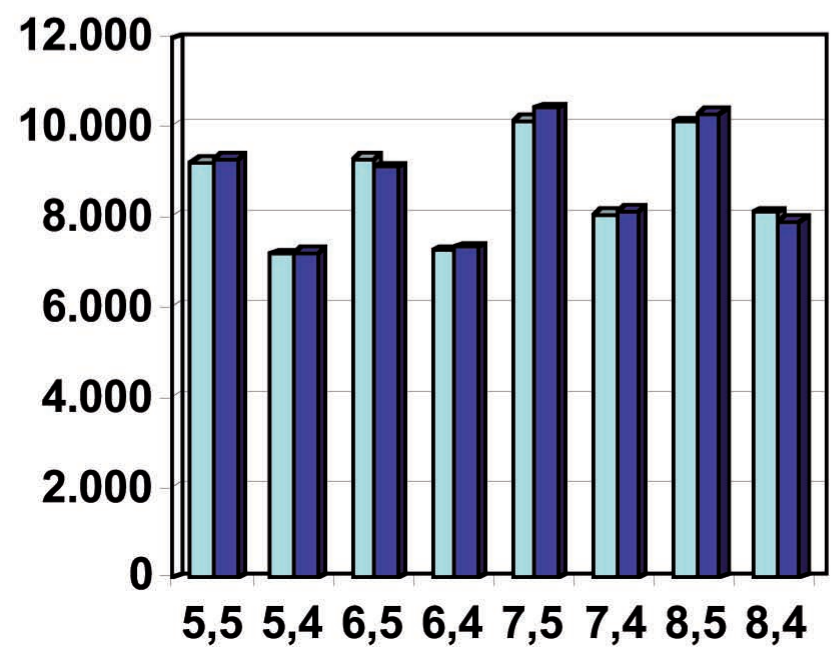

Fig. 3. Comparación de anchos mesiodistales de molares temporales según sexo.

\section{$\square$ Arco Superior \\ $\square$ Arco Inferior}


TORRES, C. M. F.: BRAVO, R. L. L.: FIERRO, M. C. \& PÉREZ, F. M. A. Anchos mesiodistales de dentición temporal en niños de la ciudad de Concepción, Chile y su correlación con el tamaño de coronas metálicas preformadas de stock. Int. J. Odontostomat., 4(1):53-57, 2010.

\section{DISCUSIÓN}

Se ha considerado oportuna la realización de este estudio biométrico en modelos de yeso debido a que en la literatura no se presentan diferencias estadísticamente significativas al realizar mediciones en boca y en modelos de estudio (Martínez et al., 2003).

Con respecto a los resultados, en este estudio las piezas de mayor tamaño son las del arco inferior siendo siempre la de mayor tamaño el $2^{\circ}$ Molar para ambos arcos, situación que se repite en otras poblaciones (Redondo \& Barbería, 2008; Martínez et al.; Kondo \& Townsed, 2005).

Al evaluar la similitud entre los molares homólogos, idealmente las piezas dentarias de un lado debieran ser del mismo tamaño que los del contralateral, así como un espejo, ya que la información genética es la misma (Kuswuandari \& Nishino, 2004). Al encontrar diferencias entre un lado y otro, éstas pueden haberse producido por factores extrínsecos o intrínsicos que usualmente reciben el nombre de "estrés". Se reportan asimetrías en poblaciones negras sudafricanas donde puede ser que una alta tasa de malnutrición participe en esta anomalía. Estos "estrés" pueden tener lugar en el período prenatal ya que las piezas temporales comienzan su mineralización en la semana 14 de gestación y las coronas recién están calcificadas al nacer. Las diferencias observadas entre las hemiarcadas no son consistentes y podemos hablar de simetrías bilaterales tanto para hombres como para mujeres. Nuestros resultados están en concordancia con los análisis realizados sobre otras poblaciones en relación a este aspecto, ya que muestran simetría en el diámetro mesiodistal (Kondo \& Townsed).

El presente estudio reveló diferencias de 0,79 $\mathrm{mm}$ en promedio al comparar anchos mesiodistales entre hombres y mujeres, es decir hay diferencias en relación al sexo pero no podemos hablar de dimorfismo sexual ya que las diferencias no son tan marcadas como en las piezas permanentes, lo que concuerda con otros estudios previos (Arnet, 2005; Jensen et al.; Redondo \& Barbería).

Tras el análisis realizado para determinar la concordancia con las coronas preformadas, podemos citar que en relación a molares maxilares existe concordancia con un tamaño determinado de corona preformada. Sólo la pieza 5.5, presentó concordancia con dos tamaños de coronas preformadas $\left(\mathrm{N}^{\circ} 2\right.$ y 3 ). En el caso de los molares mandibulares, también se puede señalar que existe concordancia con un solo tamaño de coronas preformadas $\left(\mathrm{N}^{\circ} 4\right)$; salvo la pieza 8.5 que presenta una alta concordancia con dos tamaños de coronas preformadas ( $\mathrm{N}^{\circ} 4$ y 5$)$

El ancho mesiodistal por si solo no es suficiente para comparar diámetros dentarios en diferentes poblaciones, son necesarias por lo menos tres dimensiones, el ancho mesiodistal, el bucolingual y el cervicooclusal por lo que este estudio debe desarrollarse en ése ámbito para poder efectuar comparaciones y así obtener mayores conclusiones.

Cabe señalar que la importancia clínica de estos hallazgos radica principalmente en que nos permitirá una mejor selección del tamaño adecuado de la corona metálica para cada molar temporal, ya que el ancho mesiodistal es el valor empleado como referencia principal para determinar el tamaño de la corona metálica preformada (Jensen et al.).

En este estudio se pudieran presentar sesgos a nivel de las mediciones tanto de los molares temporales en modelos de yeso, como de las mediciones de las coronas metálicas. A pesar que las mediciones fueron realizadas en modelos de estudio que aportan mayor certeza debido a la ausencia de factores externos como las mejillas, lengua, cooperación del paciente y al acceso y visibilidad propiamente tal (Redondo \& Barbería). Una forma que conciben los autores de minimizar los sesgos es utilizar modelos de yeso de mejor calidad y aparatos de medición más exactos como el pie de metro digital (Astete et al., 2009).

El presente estudio identifica anchos mesiodistales de Molares en dentición temporal pura en población infantil actual de la comuna de Concepción Chile, no encontrando diferencias estadísticamente significativas en relación al género. Se observan simetrías bilaterales en ambas arcadas.

Al analizar piezas antagonista, se observan mayores diámetros mesiodistales en el arco inferior. El promedio de ancho mesiodistal de molares temporales se corresponde de manera significativa con un solo número de corona metálica preformada de acero inoxidable 3M ESPE., salvo en las piezas $5.5 \mathrm{y}$ 8.5 , ambas del lado derecho, que se correlacionan con dos número de coronas metálicas preformadas. 
TORRES, C. M. F.: BRAVO, R. L. L.: FIERRO, M. C. \& PÉREZ, F. M. A. Anchos mesiodistales de dentición temporal en niños de la ciudad de Concepción, Chile y su correlación con el tamaño de coronas metálicas preformadas de stock. Int. J. Odontostomat., 4(1):53-57, 2010.

TORRES, C. M. F.: BRAVO, R. L. L.: FIERRO, M. C. \& PÉREZ, F. M. A. Mesiodistal width in temporary molars of Chilean children of Concepción and his correlation with standard preformed steel crowns. Int. J. Odontostomat., 4(1):53-57, 2010.

ABSTRACT: Metallic preformed steel crowns are the best treatment choice to restaurate extense cavities in temporary molars. To achieve optimal results it is necessary to know the mesiodistal width of the temporary teeth involved. We currently use other population's data regarding mesiodistal width, and since we believe there could be significant variations in between populations we decided to study mesiodistal width in our patients. The purpose of this investigation was to determine mesiodistal widths of healthy temporary molars in Chilean children. Sexual dimorphism, interarch symmetry and with the contralateral tooth, and comparison with the standard preformed steel crown were analyzed. This is a descriptive odonthometric study. 34 children (17 male, 17 female) assisting to the Pediatric Clinic were selected. Mesiodistal widths were assessed with an orthodontic caliper on a plaster model obtained from each patient. Simultaneously, preformed steel crown mesiodistal widths were measured. Results and conclusions: There were no statistically significant differences between right and left teeth's mesiodistal width, nor between genders. The mean mesiodistal width of temporary molars corresponds with only one size of preformed steel crown, except on 5.5 and 8.5. In these teeth the width corresponds with two sizes of the preformed steel crown.

KEY WORDS: mesiodistal width, temporary molars, preformed steel crown.

\section{REFERENCIAS BIBLIOGRÁFICAS}

Astete, J. C.; San pedro,V. J. \& Suazo, G. I. Sexual dimorphism in tooth dimension of Spanish and Chilean peoples. Int. J. Odontostomat., 3(1):47-50, 2009.

Arnet, A. A. Dentition and occlusion development in African American Children: mesiodistal crown diameters and tooth-size ratios of primary teeth. Pediatr. Dent., 27(2):121-8, 2005.

Jensen, E.; Kai-Jen Yen, P.; Moorrees, C. F. \& Thomsen, S. O. Mesiodistal crown diameters of the deciduous and permanent teeth in individuals. $J$. Dent. Res., 36(1):39-47, 1957.

Kondo, S. \& Townsed, G. C. Sexual dimorphism in crown units of mandibular deciduos and permanent molars in Australian Aborigines. Homo, 55(1-2):5364, 2005.

Kuswandari, S. \& Nishino, M. The mesiodistal crown diameters of primary dentition in Indonesian Javanese children. Arch. Oral Biol., 49(3):217-22, 2004

Martínez, A.; Ostos, M. J.; García, C. \& Pérez, L. Estudio de los tamaños mesiodistales en una población con dentición mixta y sus sucesores permanentes. Rev. Eur. Odonto-Estomatol., XV(4):193-200, 2003.
Ministerio de Salud. Guía Clínica Salud Oral Integral en Niños de 6 años. 1a Ed. Santiago, Ministerio de Salud de Chile, 2005

Moorrees, C. F. \& Reed, R. B. Correlation among crown diameters of human teeth. Arch. Oral Biol., 115:68597, 1964.

Randall, R. C. Preformed metal crowns for primary and permanent molar teeth: review of the literature. Pediatr. Dent., 24(5):489-500, 2002.

Redondo, A. \& Barbería, E. Tamaño mesiodistal, vestibulolingual y altura de los primeros molares permanentes de una población española. Correlación con los tamaños de las coronas preformadas utilizadas para su restauración. Gaceta dental: industria y profesiones, 188:108-29, 2008.

Dirección para correspondencia:

Dra. Francisca Torres Ch

Depto. Pediatría Bucal

Facultad de Odontología

Universidad de Concepción

CHILE

Tel: 041-2204292 - 0412207200

Email:mtorresc@udec.cl

Recibido : 09-12-2010

Aceptado: 12-03-2010 
